\title{
Computed Spectral and Temporal Emissions from a Sonoluminescing Bubble
}

\author{
W.C. Moss \\ J.A. Harte \\ J.L. Levatin \\ P.W. Rambo \\ D.A. Young \\ G.B. Zimmerman \\ I.H. Zimmerman
}

This paper was prepared for submittal to the Joint 16th International Congress on Acoustics and 135th Meeting of the Acoustical Society of America Seattle, WA

June 20-26, 1998

\section{February 1998}

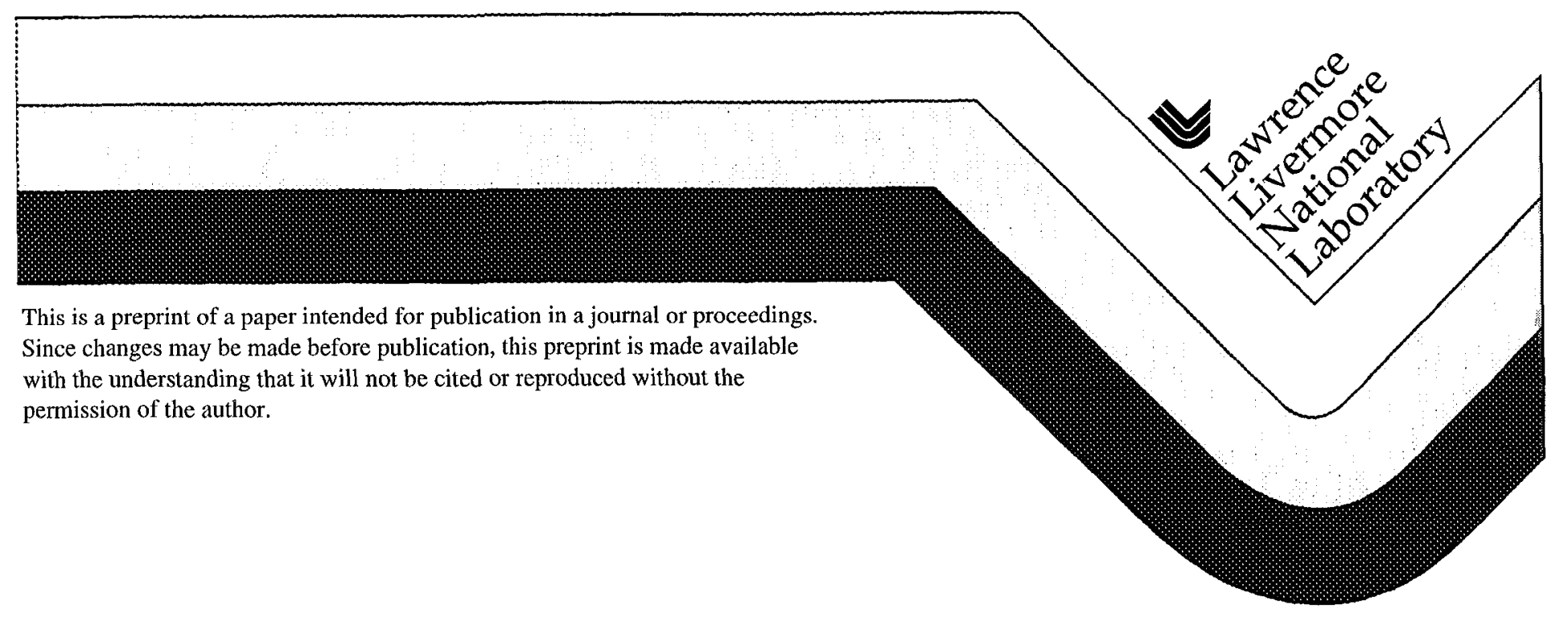




\section{DISCLAIMER}

This document was prepared as an account of work sponsored by an agency of the United States Government. Neither the United States Government nor the University of California nor any of their employees, makes any warranty, express or implied, or assumes any legal liability or responsibility for the accuracy, completeness, or uscfulness of any information, apparatus, producl, or process disclosed, or represents that its use would not infringe privately owned rights Reference herein to any specific commercial product, process, or service by trade name, trademark, manufacturer, or otherwise, does not necessarily constitute or imply its endorsement, recommendation, or favoring by the United States Government or the University of California The views and opinions of authors expressed herein do not necessarily state or reflect those of the United States Government or the University of California, and shall not be used for advertising or product endorsement purposes 


\title{
Computed spectral and temporal emissions from a sonoluminescing bubble
}

\author{
W. C. Moss, J. A. Harte, J. L. Levatin, P. W. Rambo, D. A. Young, G. B. Zimmerman, and I. H. Zimmerman
}

Lawrence Livermore National Laboratory, Livermore, CA 94550

\begin{abstract}
A sonoluminescing bubble has been modeled as a thermally conducting, partially ionized, two-component radiating plasma. The recent measurements of $\sim 100$ ps pulse widths by Gompf et al. are useful for constructing a consistent plasma thermal conduction model that refines our previous model. Normal thermal conduction in the gas and liquid, vapor pressure, and temperature-dependent surface tension arc also included in our nonlinear hydrodynamic simulations of the growth and collapse of a gas bubble and the liquid that surrounds it. We validate the model by comparing our simulations with the experimental data of Gaitan and Holt $\left[R_{o}, R_{\max }\right.$, and intensity, for different driving pressures]. We also calculate the 2D collapse of a sonoluminescing bubble in a uniform magnetic field. The numerical results show that the magnetic field induces an aspherical collapse that reduces the peak temperature in the gas and quenches SBSL. The calculated quenching is consistent with experimental data.
\end{abstract}

\section{INTRODUCTION}

Recent measurements of the light pulses emitted by single bubble sonoluminescence (1) (SBSL) show that the pulse widths may be longer than the values inferred from the original UCLA experiments (2) and can be as long as 300ps. These new results provide information for improving the modeling of SBSL. Although the mechanism of emission from a sonoluminescing bubble has still not been identified conclusively, the "plasma" model (3) appears to explain more features of SBSL than other models, including the dependence of SBSL intensity on the temperature of the water surrounding the bubble (4).

According to the plasma model (3), SBSL emission arises as follows: As the bubble collapses, the contents of the bubble are compressed and heated. The hotter regions begin to ionize and create a two-component plasma of ions and electrons, e.g., we previously calculated the density and temperature of the radiating plasma of a sonoluminescing air bubble to be $\sim 1 \mathrm{~g} / \mathrm{cc}$ and $\sim 55000 \mathrm{~K}$. This "cool" dense plasma emits light by an energy cascade from the ions, to the electrons, to the photons. The electron-photon coupling can be due to bremsstrahlung, Compton scattering, photoionization, and/or line transitions. However, for what we believe to be typical SBSL conditions, bremsstrahlung from thermally excited electrons is probably the dominant mechanism. The energy of the emitted light is very small compared to the thermal energy of the ions and electrons, so the pulse width is governed by energy losses from the ions and electrons. The main loss mechanism is plasma thermal conduction, which occurs at very different rates in the ions $\left(\sim T_{i}^{5 / 2} M_{i}^{-1 / 2}\right)$ and electrons $\left(\sim T_{c}^{5 / 2} M_{e}^{-1 / 2}\right)$, due primarily to the large difference between the ion and electron masses $M_{i}$ and $M_{e}$. (The ion-electron collision times are extremely short [ $\sim \mathrm{fs}$ ], much shorter than the optical emission time, so the ion and electron temperatures $T_{i}$ and $T_{e}$ are nearly identical in the emitting region of the bubble.) Consequently, electron conduction is the dominant loss mechanism, and the strong temperature dependence of the conductivity ( $5 / 2$ exponent) is responsible for producing a short pulse width. We note parenthetically that if the light is thermal in origin, then "sonoluminescence" is a misnomer; sonoincandescence is more appropriate.

\section{MODEL AND RESULTS}

We showed in our earlier work (3) that SBSL intensity was very sensitive to the ambient bubble radius $R_{0}$ and the maximum bubble radius $R_{\max }$, but the literature contained no simultaneous measurements of $R_{\varphi}, R_{\max }$, and SBSL intensity, which along with spectra, are required to do accurate modeling of a particular experiment. Thus we included in our modeling only those physical mechanisms that we believed had a primary effect on the energetics of the collapse. Recent measurements of the dependence of $R_{0}, R_{\max }$, and SBSL intensity on driving pressure (6) provide more constraints for our modeling, so we have added additional physics to improve the accuracy of our calculations. Specifically, we include vapor pressure, normal thermal conduction (in addition to plasma thermal conduction), and a temperature dependent surface tension in our nonlinear, compressible hydrodynamic simulations of the growth and collapse of a gas bubble and the liquid that surrounds it.

We have also modified the plasma thermal conduction model, in light of the new longer pulse width data. The short 
pulse widths that were reported originally implied that a very fast mechanism, such as "normal" plasma thermal conduction $\left(\sim \mathrm{T}^{5 / 2}\right)$ was responsible for removing energy from the bubble. Coincident with the reporting of the longer pulse widths, F. MacIntyre made the observation that the SBSL plasma has a very small Debye screening length (5), which means that the electrons are close to the ions and almost completely screen the ionic electric fields. Consequently, the plasma behaves like a typical dense fluid with two-components (ions and electrons) whose transport properties arise from collisional rather than coulombic interactions. This type of plasma is called a "strongly coupled plasma." The main difference between this strongly coupled plasma and the "normal" plasma described earlier is that the rate of energy loss by plasma thermal conduction is much less in the strongly coupled plasma $\left(\sim T^{1 / 2}\right.$ for both ions and electrons), which gives rise to longer and variable calculated pulse widths that are consistent with the new experimental measurements.

We note that these refinements in our model do not change the qualitative results of our previous work. They improve the quantitative results and our predictive capability. We will demonstrate this by showing comparisons of our SBSL numerical simulations and recent experimental data $(1,6)$. We will also show results of 2D simulations of SBSL in a uniform magnetic field. Experimental data show that SBSL is quenched by a sufficiently large magnetic field (7). Our calculations show that the magnetic field induces an aspherical collapse that reduces the peak temperature in the gas and quenches SBSL. The calculated quenching is consistent with experimental data.

\section{ACKNOWLEDGMENTS}

WCM thanks D. Boercker, F. Rogers, and C. Iglesias for discussions about plasmas, C. McMillan for providing IHZ's time, and N. Burkhard for his continuing support. This work was performed under the auspices of the U. S. Department of Energy by Lawrence Livermore National Laboratory under Contract No. W-7405-Eng-48.

\section{REFERENCES}

1 Gompt, B. et al., Phys. Rev. Lett. 79, 1405-1408 (1997)

2 Barber, B. P. and Putterman, S J., Nature 352, 318-320 (1991).

3 Moss, W C, Clarke, D B , and Young, D A., Science 276, 1398-1401 (1997)

4 Hilgenfeldt, S., Lohse, D, and Moss, W C, Phys Rev. Lett 80, 1332-1335 (1998).

5 Macintyre, F, presentation at Nato Advanced Studies Institute, Leavenworth, WA (1997)

6 Gaitan, D F and Holt, R G., unpublished data (1997)

7 Young, J. B., Schmiedel, T, and Kang, W, Phys. Rev. Lett 77, 4816-4819 (1996). 


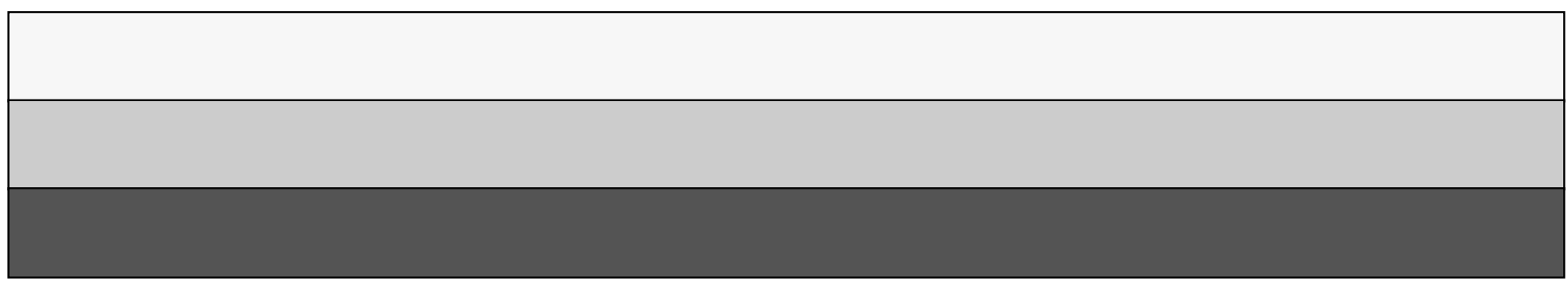

